\title{
Changes in aortic collagen in $\beta$-aminopropionitrile-induced acute aortic dissection
}

\author{
Hao Zhou ${ }^{1,2,3} \wedge$, Yan Ren ${ }^{4}$, Jun Xiao ${ }^{1,2,3}$, Jian $\mathrm{He}^{1,2,3}$, Yuling Zhang ${ }^{1,2,3}$, Zhihuang Qiu ${ }^{1,2,3}$, Qiuyu Huang ${ }^{1,2,3}$, \\ Yunnan $\mathrm{Hu}^{1,2,3}$, Liangwan Chen ${ }^{1,2,3}$ \\ ${ }^{1}$ Department of Cardiac Surgery, Fujian Medical University Union Hospital, Fuzhou, China; ${ }^{2}$ Key Laboratory of Ministry of Education for \\ Gastrointestinal Cancer, School of Basic Medical Sciences, Fujian Medical University, Fuzhou, China; ${ }^{3}$ Fujian Key Laboratory of Cardio-Thoracic \\ Surgery (Fujian Medical University), Fuzhou, China; ${ }^{4}$ Department of Cardiac Surgery, Zunyi Medical University Affiliated Hospital, Zunyi, China \\ Contributions: (I) Conception and design: H Zhou, L Chen; (II) Administrative support: L Chen, Q Huang; (III) Provision of study materials or \\ patients: J Xiao, J He, L Chen; (IV) Collection and assembly of data: Y Ren, Y Hu, H Zhou; (V) Data analysis and interpretation: H Zhou, Z Qiu, L \\ Chen; (VI) Manuscript writing: All authors; (VII) Final approval of manuscript: All authors. \\ Correspondence to: Liangwan Chen, MD. Department of Cardiac Surgery, Fujian Medical University Union Hospital, Xinquan Road No. 29, Fuzhou \\ 350001, China. Email: chenliangwan@tom.com.
}

Background: The precise role collagen plays in acute aortic dissection (AAD) was investigated in an animal model of $\beta$-aminopropinitrile (BAPN)-induced AAD.

Methods: The 30 3-week-old male specific-pathogen free (SPF)-grade Sprague-Dawley (SD) rats were randomly divided into two groups: 10 in the Control group and 20 in the Model group. The Model group was treated with $0.1 \%$ BAPN for 4 weeks, while the Control group received untreated water. Histopathological staining and western blot were used to detect changes of the extracellular matrix (ECM) and collagen content in the aorta.

Results: At the end of the experiment, the incidence of AAD was $25 \%$, the aortic ECM of surviving rats was severely damaged, and the arrangement was disordered. Fibroblast cells are unevenly distributed, with wide gaps, collagen fibers were also distributed unevenly in a disordered arrangement and their thickness was uneven. The elastic membrane disappeared over a large area. Compare to Control group, the Collagen types I, $\mathrm{III}$ and their subunits were upregulated $(\mathrm{P}<0.05)$, while matrix metalloproteinase (MMP) 2 and MMP9 were downregulated in the aorta of Model group $(\mathrm{P}<0.05)$.

Conclusions: In the animal model of BAPN-induced AAD, collagen types I, III and subunits were increased, while MMP2 and MMP9 were decreased in thoracic aorta, which may lead to stiffness of the aorta and be the cause of dissection.

Keywords: Acute aortic dissection (AAD); animal models; $\beta$-aminopropinitrile (BAPN); collagen; matrix metalloproteinase

Submitted Sep 01, 2021. Accepted for publication Oct 16, 2021.

doi: $10.21037 /$ atm-21-4933

View this article at: https://dx.doi.org/10.21037/atm-21-4933

\section{Introduction}

Acute aortic dissection (AAD) refers to rupture of the aortic intima and is a disastrous condition, but the precise mechanism is currently unknown. Although there has been great progress in dealing with $\mathrm{AAD}$, the surgical mortality rate is still high $(1,2)$.

^ ORCID: 0000-0001-8019-0913. 
The extracellular matrix (ECM) determines the structure of the blood vessel wall and is mainly composed of collagen and elastin. On the one hand, collagen provides antimechanical tension, but on the other hand, it plays an important role in maintaining the normal physiological process of blood vessels. Collagen plays different roles in the occurrence and development of aortic diseases (3). One study has shown that type I and type III collagen are significantly increased in thoracic $\mathrm{AD}$ in human, the authors therefore inferring that vasodilation and contraction compliance are compromised and ultimately participate in the occurrence of the dissection (4). However, another study pointed out that in ascending aortic aneurysms and dissections, collagen content is reduced, and the subsequent weakening of the blood vessel wall may be the basis of the disease (5). One research has reported that in BAPN induced $\mathrm{AAD}$ in mice the mRNA expression of collagen type I alpha 2 (Col1a2), collagen type IV alpha4 (Col4a4) were decreased (6).

$\mathrm{AAD}$ are associated with degenerative changes in the medial layer of the aortic wall. Matrix metalloproteinases (MMPs) are a family of proteolytic enzymes that degrade various components of the ECM and play an important role in vascular remodeling. Dysregulation of MMP production and activity can lead to the happening of AAD (7).

At present, the classical method of inducing an animal model of $\mathrm{AAD}$ is treating mice or rats with $\beta$-aminopropinitrile (BAPN) combined with angiotensin II (AngII), and the incidence of AAD is approximately $100 \%$ (8). Based on our previous experiment (9), we have refined the establishment of the animal model of BAPN-induced AAD, which we used in the current study to investigate any changes in aortic collagen content to understand the precise mechanism and role of collagen in human AAD.

We present the following article in accordance with the ARRIVE reporting checklist (available at https://dx.doi. org/10.21037/atm-21-4933).

\section{Methods}

\section{Materials}

\section{Experimental animals}

We obtained 30 healthy SPF-grade, 3-week-old male SD rats weighing $51.3 \pm 4.5 \mathrm{~g}$ from Shanghai Slack Laboratory Animal Co., Ltd. [Production License No. SCXK (Hu) 2017-0005]. The animals were kept at $22-25^{\circ} \mathrm{C}$ with an alternating light-dark cycle $(12 / 12 \mathrm{~h})$. The rats ate and drank according to the experimental design. Experiments were performed under a project license (No. FJMU IACUC 2019-0026.) granted by Experimental Animal Care and Use Committee of Fujian Medical University, in compliance with Fujian Medical University institutional guidelines for the care and use of animals. A protocol was prepared before the study without registration.

\section{Main drugs and reagents}

BAPN was purchased from TCI (Japan); rabbit monoclonal anti-MMP9 antibody (ab76003), rabbit monoclonal antiMMP2 antibody (ab92536), and rabbit monoclonal anticollagen I antibody (ab260043) were purchased from Abcam (UK); rabbit anti-COL1 $\alpha 1$ (A16891), COL1 $\alpha 2$ (A16699), and COL3a1 (A3795) were purchased from Abclonal (China); rabbit anti-collagen III antibody (22734-1-AP) was purchased from Sanying Biotechnology (China).

\section{Study methods}

\section{Animal model}

After 3 days' acclimatization before the experiment, the SD rats were randomly divided into two groups: 10 in the Control group and 20 in the Model group. In the Control group, the rats were fed commercial feed pellets and provided with autoclaved water for drinking throughout the study. In the Model group, BAPN at a final concentration of $0.1 \%$ was added to the drinking water $(0.2 \mathrm{~mL}$ BAPN $+200 \mathrm{~mL}$ water) (10), which was protected from light to prevent the decomposition of BAPN. Body weight was measured every 2 days, and any rats that died before the end of the study period were autopsied to confirm the presence of aortic dissection, if the AAD was formed, the aorta were harvested and were defined as aorta ruptured group. At the end of 4 weeks, the surviving rats were anesthetized with $2 \%$ pentobarbital sodium by intraperitoneal injection and the ascending to descending aorta was harvested, as had been excised from the rats that died before. Unless otherwise specified, the samples used in the Model group were from aorta free of AAD (aorta unruptured group). In our previous study (9), the rats were treated with BAPN combined with AngII, a potent vasoconstrictor, and the incidence of AAD was approximately $90 \%$. Once the AAD occurs, there is a severe stress response, the structure of the aorta is destroyed, and blood cells infiltrate the aortic wall, which affects the results of the test. In fact, most cases of AAD occurred after AngII was injected, which suggested that before AngII was injected, the aorta of the rats had already 


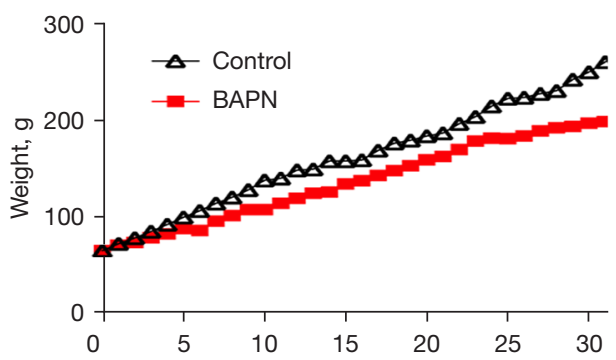

Figure 1 Weight gain in the Control group and Model group during the experimental period. The average weight of the Model group was significantly lower than that of the Control group at the end of the experiment, $\mathrm{P}<0.05$.

undergone pathological change, so in the present study, we treated the rats with BAPN only.

\section{Histopathological analysis}

\section{Scanning electron microscopy (SEM)}

Briefly, fresh aorta tissues were harvested, and immediately fixed with electron microscopy fixative (Servicebio, China) for $2 \mathrm{~h}$ at room temperature. The tissue blocks were washed three times with $0.1 \mathrm{M}$ phosphate-buffered saline (PBS) ( $\mathrm{pH}$ 7.4) for $15 \mathrm{~min}$ each time. The tissue blocks were then transferred into $1 \% \mathrm{OsO}_{4}$ in $0.1 \mathrm{M}$ PBS (pH 7.4) for $1-2 \mathrm{~h}$ at room temperature. Next, the tissue blocks were again washed three times with $0.1 \mathrm{M}$ PBS ( $\mathrm{pH} 7.4$ ) for $15 \mathrm{~min}$ each time. The samples then were dehydrated and dried by ethanol and Critical Point Dryer respectively. Specimens were attached to metallic stubs using carbon stickers and spatter-coated with gold for $30 \mathrm{~s}$. Images were taken immediately with SEM (HITACHI SU8100).

\section{Tissue staining}

The aortic tissues were paraffin-embedded, cut at 5- $\mu \mathrm{m}$ thickness and stained with hematoxylin-eosin (H\&E), Masson Trichrome, and elastica van Gieson (EVG). Image analysis was performed using Image-J software.

\section{Western blot analysis}

The ascending and descending aorta were frozen in liquid nitrogen and stored at $-80{ }^{\circ} \mathrm{C}$ for preservation. Protein was extracted using a commercial kit. Equal amounts of protein $(30 \mu \mathrm{g} /$ lane $)$ were separated using $4-12 \%$ SDSPAGE electrophoresis. The blots were probed with primary antibodies, anti-GAPDH $(1: 1,000)$, anti-MMP2 $(1: 1,000)$, anti-MMP9 $(1: 1,000)$, anti-COL1 $\alpha 1 \quad(1: 1,000)$, antiCOL1 $\alpha 2(1: 1,000)$, anti-COL3 $\alpha 1$ (1:1,000), anti-collagen
I $(1: 1,000)$, and anti-collagen III $(1: 500)$ for $16 \mathrm{~h}$ at $4{ }^{\circ} \mathrm{C}$, followed by HRP-conjugated secondary antibody $(1: 10,000)$ for $1 \mathrm{~h}$. Immunoblotting was performed according to the manufacturer's guidelines (Bio/Rad, Hercules, CA, USA). Densitometry analysis was performed using Image-J software.

\section{Statistical analysis}

The results from at least three independent experiments were used to calculate mean $\pm \mathrm{SD}$, Student's $t$-test was used for statistical analysis by Prism8, and $\mathrm{P}<0.05$ was considered statistically significant.

\section{Results}

\section{General condition of rats given BAPN treatment}

There was no statistical difference of body weight in each group $(\mathrm{P}>0.05)$ at the beginning of the experiment. The rats in the Control group were in good condition throughout the experiment, and their weight gradually increased, whereas the weight of the Model group gradually lagged behind the Control group after drinking BAPN for 3 days. The weight of the Model group was significantly lower than that of the Control group at the end of the experiment, $\mathrm{P}<0.05$ (Figure 1).

\section{BAPN-induced AAD}

In the Model group, AAD began to occur at approximately 22 days after BAPN administration (Figure 2). Ruptures were mostly located at the side of the lesser curvature of the aortic arch, and the ascending aorta was mostly involved. An intimal flap formed, dividing the blood vessel into true and false cavities (Figure 3), appearing similar to human aortic dissection. At the end of the experiment the incidence of AAD was $25 \%(\mathrm{n}=5) ; 15$ rats survived.

\section{ECM change after BAPN administration}

Collagen and elastin are the major structural proteins of the aortic wall. In collagen, cross-linkages bind the collagen molecules and fibers together and are essential in providing the tensile strength and mechanical stability of the collagen fibrils and fibers (11). BAPN, a lysyloxidase (LOX) inhibitor, irreversibly inhibits the formation of crosslinkages in collagen (12).

After BAPN administration, SEM of the aorta showed that in the Control group smooth muscle cells (SMC) 


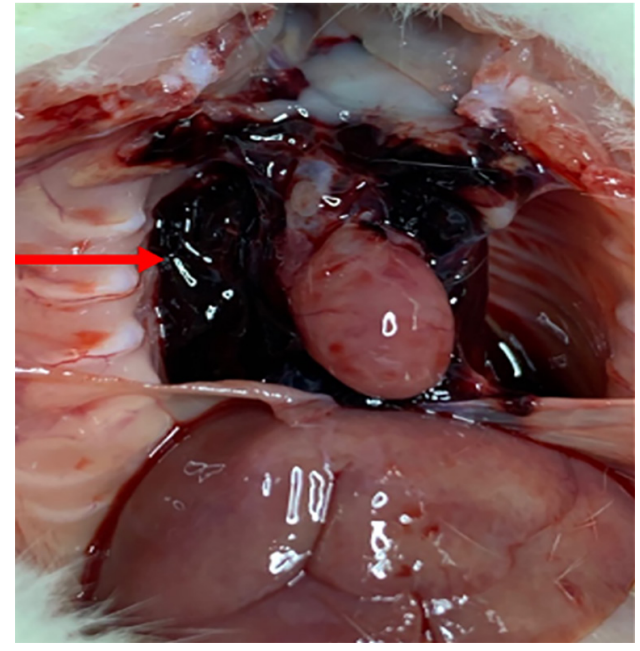

Figure 2 Large amounts of hemorrhage and blood clots (the red arrow) in the thoracic cavity of rats when the aorta ruptured.

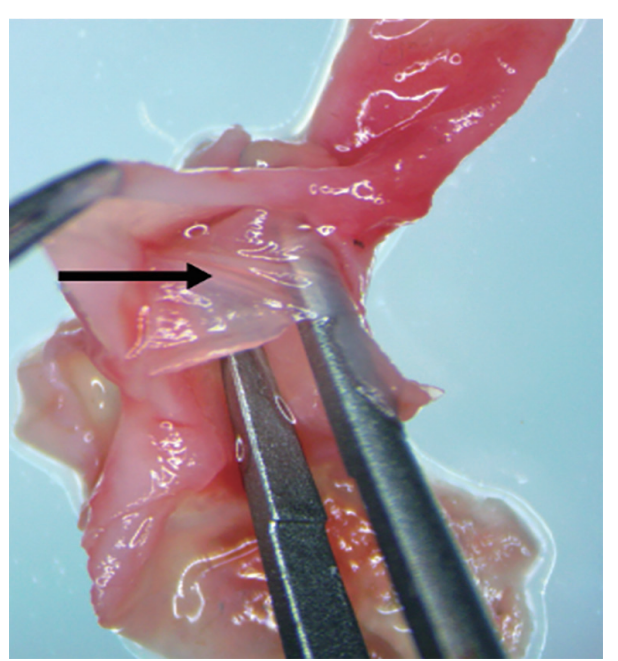

Figure 3 Example of acute aortic dissection involving the ascending aorta. An intimal flap (arrow) has formed, dividing the vessel into true and false lumens.

were evenly distributed, with normal collagen fibers of uniform thickness evenly distributed among them. The elastic membrane had normal continuous structure, uniform thickness, and parallel arrangement. In the Model group, the structure of the aortic media layer was severely damaged, and the arrangement was disordered. Fibroblast cells are unevenly distributed, with wide gaps, collagen fibers were also distributed unevenly in a disordered arrangement and their thickness was uneven. The elastic membrane disappeared over a large area (Figure 4).

\section{Histopathological staining}

HE staining shown that in the Control group, the structure of the blood vessel wall was complete, with even distribution of SMCs. In the Model group, the vascular wall was thickened (Figure 5). EVG staining shown the elastin fibers were thinning, disappeared and arranged irregularly, while Masson trichrome staining shown the collagen fibers were significantly increased and thickened (Figure 6).

\section{Western blot test and expression of MMPs}

Compared with the Control group, the expression of collagen type I, type III and their subunits were upregulated in the Model group $(\mathrm{P}<0.05)$ (Figure 7). The expression of MMP9 and MMP2 in the aorta was downregulated in the Model group when the aorta was free of dissection $(\mathrm{P}<0.05)$, while in the dissection formed aorta the expression was significantly higher than in the Control and aorta intact group $(\mathrm{P}<0.01)$ (Figure 8$)$.

\section{Discussion}

As one of the major components of the aortic wall, collagen plays a significant role in the blood vessel's function and mechanical properties, such as tensile strength and stiffness. Collagen molecule were composed of three polypeptide segments, namely alpha chains $(3,13,14)$ and the main types of collagen found in the aorta are types I and III. As a LOX inhibitor, BAPN irreversibly inhibits the formation of crosslinks (12), is known to induce aortic dissection in animals, and also can leads to a decrease in collagen synthesis (15). However, in this study when BAPN was given to rats, we found the protein concentrations of collagen types I and III and their subunits were increased in the thoracic aorta. The increased levels of collagen types I and III may be due to a compensatory increase of their subunits. Increased collagen content can lead to aortic stiffness, increasing the likelihood of rupture $(16,17)$, and may be the mechanism of BAPNinduced AAD.

The SEM images showed severe damage to the structure of the aortic media layer. The distribution and thickness of the collagen fibers was uneven, and their arrangement was disordered, findings that are consistent with aging- and hypertension-induced changes in the human aorta (18); $80 \%$ of $\mathrm{AD}$ patients have hypertension (19). As AAD risk 


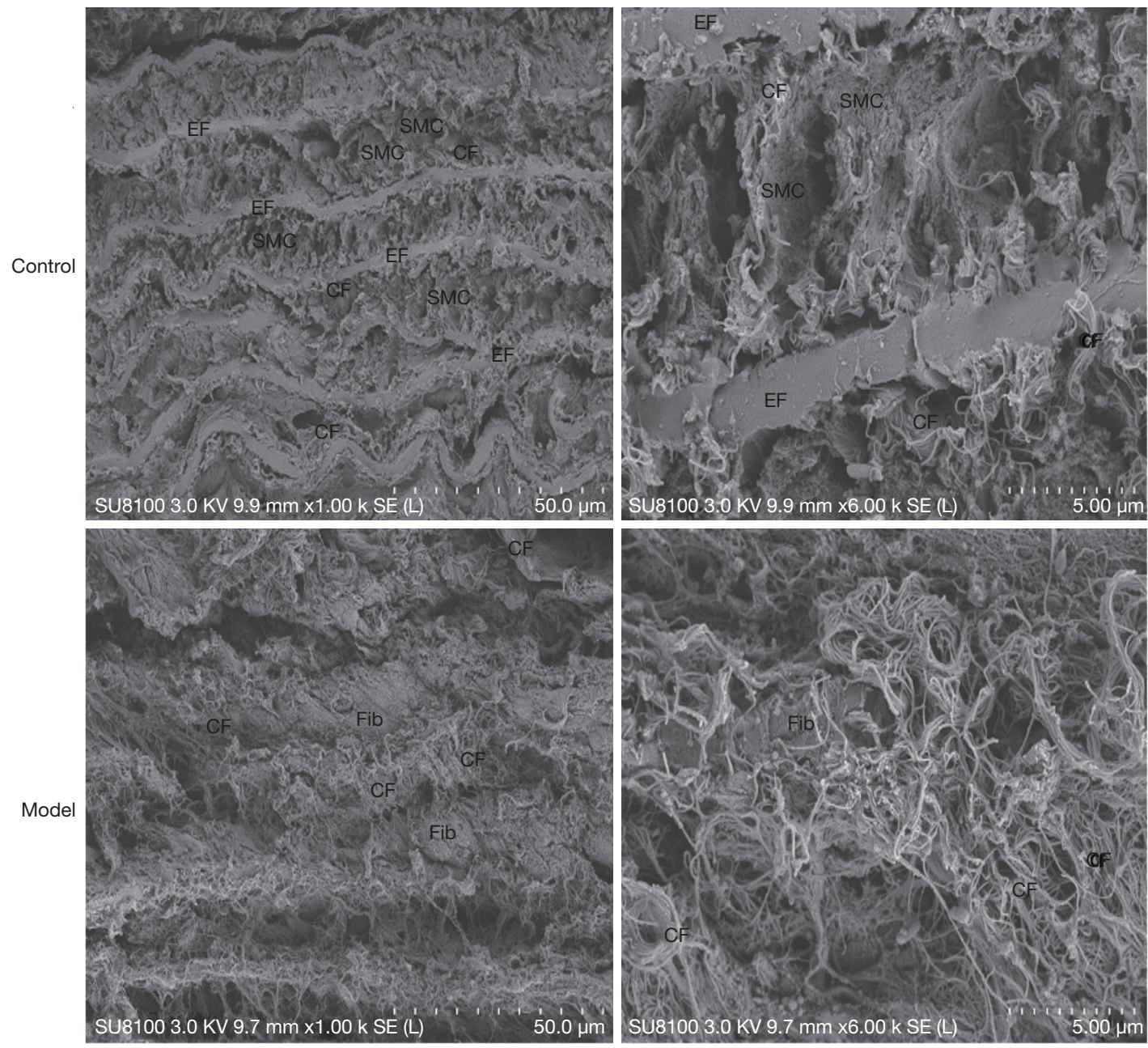

Figure 4 Scanning electron microscopy of the aorta. Compared with the Control group, the structure of the aortic media layer in the Model group is severely damaged. Fib cells show a disorder distribution with wide gaps. The distribution and thickness of CF is uneven, and their arrangement is disordered. The EF has disappeared over a large area. CF, collagen fibers; EF, elastic film; Fib, fibroblast cells; SMC, smooth muscle cell.

factors (20), aging, hypertension and smoking commonly lead to collagen deposition, increasing the stiffness of the aorta (21-23). During strenuous activities, such as weightlifting, there is a high risk of AAD, suggesting that rapid blood pressure fluctuation over a short period of time may induce AAD on the basis of already existing vascular pathological changes (24). This is similar to our observations from the AAD model in our previous study. When rats were treated with BAPN only, the incidence of $\mathrm{AAD}$ was low, but when given BAPN combined with AngII injection, the incidence of AAD significantly increased.

The relationship between the MMP family of enzymes that degrade collagen and aortic diseases has been widely studied (25). Generally, the expression of MMP2 and MMP9 is considered to be increased in AAD (26). In our present study, the concentrations of MMP2 and MMP9 in the unruptured aorta were decreased, but were increased in ruptured aorta. This contradictory phenomenon may be explained by the fact that once the aorta ruptures, a large number of blood cells infiltrate the aortic wall, and both MMP9 and MMP2 are secreted by macrophages and neutrophils. In view of the high incidence of AAD in the animal model, it infers that in the BAPN-induced AAD model, the expression of MMP2 and MMP9 in the aorta was downregulated, and matrix degradation may not participate in the occurrence of $\mathrm{AAD}$, while the 

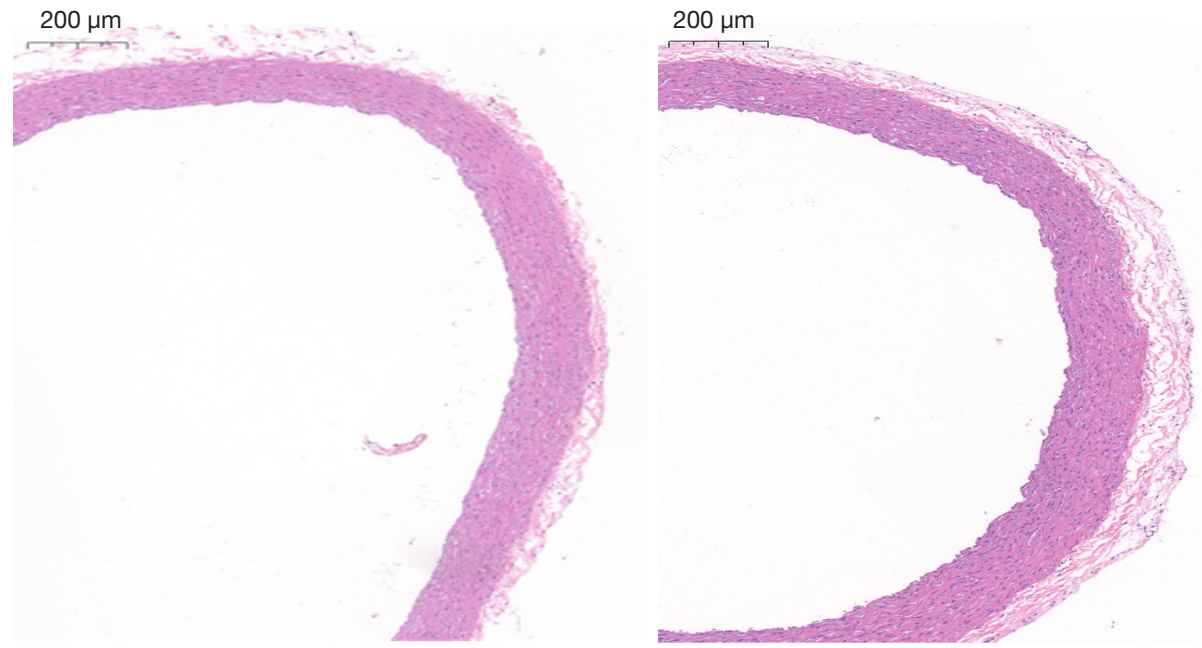

Figure $5 \mathrm{HE}$ staining of the aorta. In the Control group, the structure of the blood vessel wall is complete, the smooth muscle cells are evenly distributed, the cells are neatly arranged, and no morphological changes of tissues can be seen. The vascular wall in the Model group is thickened. Scale bar: $200 \mu \mathrm{m}$.
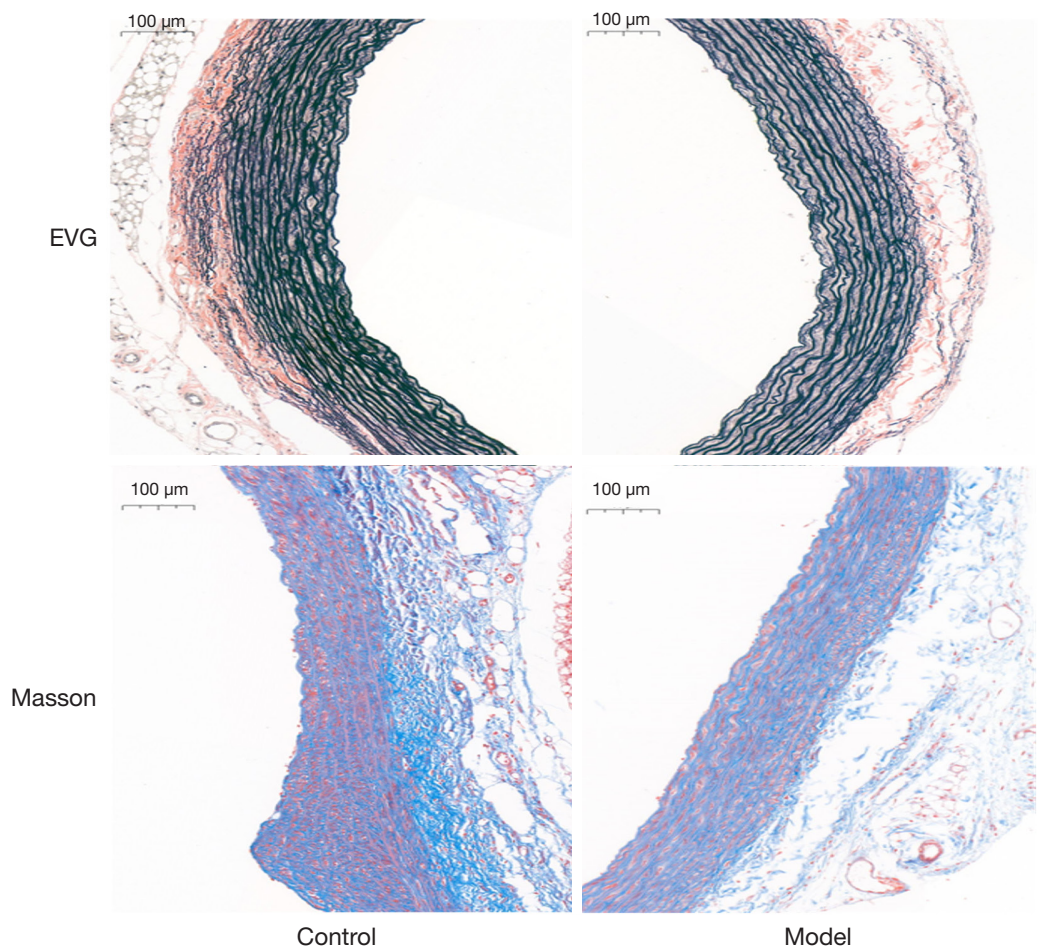

A

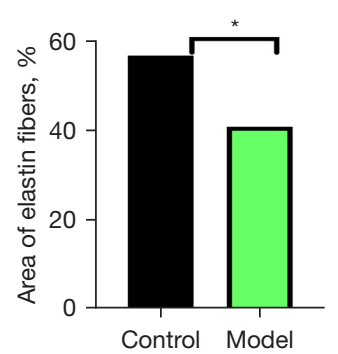

B

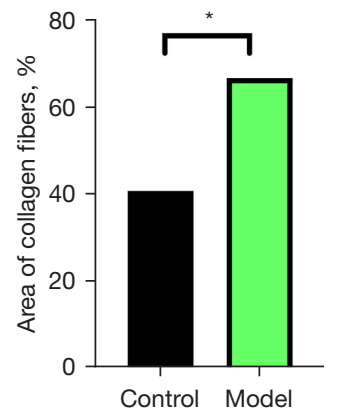

Figure 6 Pathologic evaluation of the extracellular matrix of the aorta. Elastin fibers were detected by EVG staining, collagen fibers were detected by Masson trichrome staining. Elastin fibers in the Model group were thinning, disappeared, and arranged irregularly. Collagen fibers were significantly increased and thickened. (A) Percent area of elastin fibers, (B) percent area of collagen fibers. *, P<0.05. Scale bar: $100 \mu \mathrm{m}$. EVG, elastica van Gieson. 

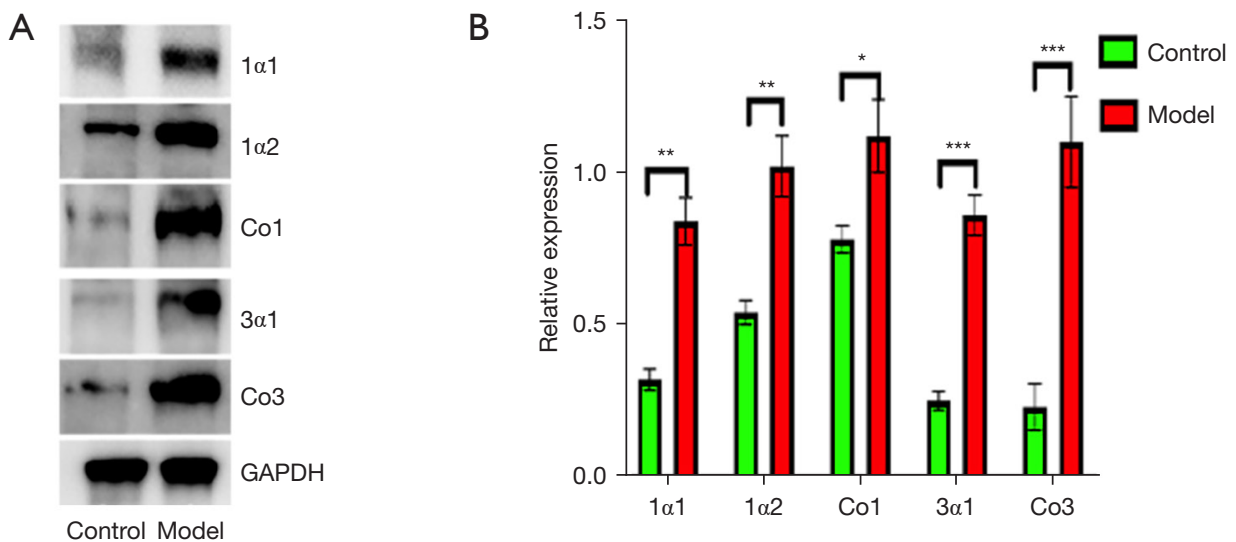

Figure 7 Collagen type I, and its subunits $1 \alpha 1,1 \alpha 2$, type III and its subunit, $3 \alpha 1$ were examined by western blot (A). The relative expression is shown in (B). *, $\mathrm{P}<0.05$. **, $\mathrm{P}<0.01$. ***, $\mathrm{P}<0.005$. MMP, matrix metalloproteinase.

A

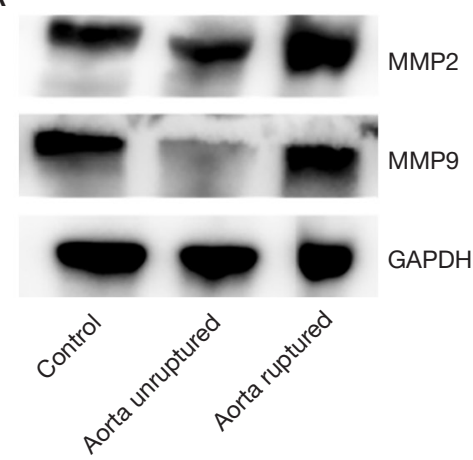

B

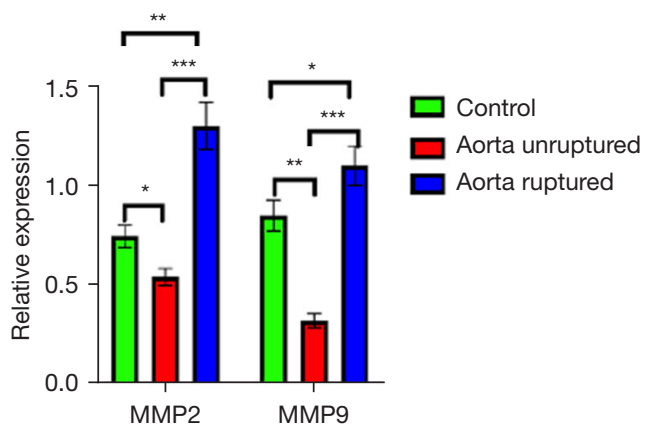

Figure 8 Expressions of MMP9 and MMP2 were downregulated in the aorta unruptured group of the Model group, but were significantly higher in the aorta ruptured group than the Control and aorta unruptured groups (A). The relative expression is shown in (B). * $\mathrm{P}<0.05$; **, $\mathrm{P}<0.01 ; * *, \mathrm{P}<0.005$. MMP, matrix metalloproteinase.

upregulation of expression in ruptured aorta was most likely associated with the blood cells.

A limitation of our study is that the BAPN-induced AAD model does not completely mimic the process of human AD. In addition, the other types of collagen in the aorta were not tested in this study. Future studies are needed to fully explore the changes of collagen in the aorta.

In conclusion, as a disastrous disease, the precise cause of the AAD is currently unknown. In this study, we demonstrated that collagen types I, III and subunits were increased in a BAPN-induced AAD model. The distribution and thickness of collagen fibers in the aortic media layer was uneven and their arrangement was disordered, which may increase the stiffness of the aorta, and predispose it to rupture. At the same time, the expressions of MMP2 and
MMP9 in aorta were decreased, which may also contribute to collagen deposition in the aorta.

\section{Acknowledgments}

Funding: This work was supported by the National Natural Science Foundation of China (U2005202), the Fujian Province Major Science and Technology Program (2018Yz0001), the Natural Science Foundation of Fujian Province (2020J01998, 2020J02056), and the Fujian Provincial Health Technology Project (2019-ZQN-50).

\section{Footnote}

Reporting Checklist: The authors have completed the 
ARRIVE reporting checklist. Available at https://dx.doi. org/10.21037/atm-21-4933

Data Sharing Statement: Available at https://dx.doi. org/10.21037/atm-21-4933

Conflicts of Interest: All authors have completed the ICMJE uniform disclosure form (available at https://dx.doi. org/10.21037/atm-21-4933). The authors have no conflicts of interest to declare.

Ethical Statement: The authors are accountable for all aspects of the work in ensuring that questions related to the accuracy or integrity of any part of the work are appropriately investigated and resolved. Experiments were performed under a project license (No. FJMU IACUC 2019-0026.) granted by Experimental Animal Care and Use Committee of Fujian Medical University, in compliance with Fujian Medical University institutional guidelines for the care and use of animals.

Open Access Statement: This is an Open Access article distributed in accordance with the Creative Commons Attribution-NonCommercial-NoDerivs 4.0 International License (CC BY-NC-ND 4.0), which permits the noncommercial replication and distribution of the article with the strict proviso that no changes or edits are made and the original work is properly cited (including links to both the formal publication through the relevant DOI and the license). See: https://creativecommons.org/licenses/by-nc-nd/4.0/.

\section{References}

1. Pape LA, Awais M, Woznicki EM, et al. Presentation, Diagnosis, and Outcomes of Acute Aortic Dissection: 17Year Trends From the International Registry of Acute Aortic Dissection. J Am Coll Cardiol 2015;66:350-8.

2. Zhu Y, Lingala B, Baiocchi M, et al. Type A Aortic Dissection-Experience Over 5 Decades: JACC Historical Breakthroughs in Perspective. J Am Coll Cardiol 2020;76:1703-13.

3. Berillis P. The Role of Collagen in the Aorta's Structure. The Open Circulation \& Vascular Journal 2013;6.

4. Wang X, LeMaire SA, Chen L, et al. Increased collagen deposition and elevated expression of connective tissue growth factor in human thoracic aortic dissection. Circulation 2006;114:I200-5.

5. de Figueiredo Borges L, Jaldin RG, Dias RR, et al.
Collagen is reduced and disrupted in human aneurysms and dissections of ascending aorta. Hum Pathol 2008;39:437-43.

6. Yyya B, Lyla B, Xlja B, et al. Intermittent Hypoxia Alleviates $\beta$-Aminopropionitrile Monofumarate Induced Thoracic Aortic Dissection in C57BL/6 Mice. Eur J Vasc Endovasc Surg 2020;59:1000-10.

7. Zhang X, Shen YH, LeMaire SA. Thoracic aortic dissection: are matrix metalloproteinases involved? Vascular 2009;17:147-57.

8. Jiang DS, Yi X, Zhu XH, et al. Experimental in vivo and ex vivo models for the study of human aortic dissection: promises and challenges. Am J Transl Res 2016;8:5125-40.

9. Lv X, Hu Y, Chen X, et al. Establishment and effect evaluation of an aortic dissection model induced by different doses of $\beta$-aminopropionitrile in rats. Vascular 2020;1708538120984056.

10. $\mathrm{Hu}$ Y, Lu L, Qiu Z, et al. Mechanical stretch aggravates aortic dissection by regulating MAPK pathway and the expression of MMP-9 and inflammation factors. Biomed Pharmacother 2018;108:1294-302.

11. Brüel A, Ortoft G, Oxlund H. Inhibition of cross-links in collagen is associated with reduced stiffness of the aorta in young rats. Atherosclerosis 1998;140:135-45.

12. Tang SS, Trackman PC, Kagan HM. Reaction of aortic lysyl oxidase with beta-aminopropionitrile. J Biol Chem 1983;258:4331-8.

13. Vouyouka AG, Pfeiffer BJ, Liem TK, et al. The role of type I collagen in aortic wall strength with a homotrimeric. J Vasc Surg 2001;33:1263-70.

14. Silver FH, Horvath I, Foran DJ. Viscoelasticity of the vessel wall: the role of collagen and elastic fibers. Crit Rev Biomed Eng 2001;29:279-301.

15. Kenyon NJ, Ward RW, McGrew G, et al. TGF-beta1 causes airway fibrosis and increased collagen I and III mRNA in mice. Thorax 2003;58:772-7.

16. Tsamis A, Stergiopulos N, Rachev A. A structure-based model of arterial remodeling in response to sustained hypertension. J Biomech Eng 2009;131:101004.

17. Farra YM, Matz J, Ramkhelawon B, et al. Structural and functional remodeling of the female Apoe-/- mouse aorta due to chronic cigarette smoke exposure. Am J Physiol Heart Circ Physiol 2021;320:H2270-82.

18. Zhang J, Zhao X, Vatner DE, et al. Extracellular Matrix Disarray as a Mechanism for Greater Abdominal Versus Thoracic Aortic Stiffness With Aging in Primates. Arterioscler Thromb Vasc Biol 2016;36:700-6.

19. Gawinecka J, Schönrath F, von Eckardstein A. Acute aortic 
dissection: pathogenesis, risk factors and diagnosis. Swiss Med Wkly 2017;147:w14489.

20. Golledge J, Eagle KA. Acute aortic dissection. Lancet 2008;372:55-66.

21. Ribeiro P, Jadhav AV, Walesby R, et al. Collagen content of atherosclerotic arteries is higher in smokers than in non-smokers. Lancet 1983;1:1070-3.

22. Babici D, Kudej RK, McNulty T, et al. Mechanisms of increased vascular stiffness down the aortic tree in aging, premenopausal female monkeys. Am J Physiol Heart Circ Physiol 2020;319:H222-34.

23. Susic D, Varagic J, Ahn J, et al. Collagen cross-link breakers: a beginning of a new era in the treatment of cardiovascular changes associated with aging, diabetes, and hypertension. Curr Drug Targets Cardiovasc Haematol Disord 2004;4:97-101.

24. Hatzaras I, Tranquilli M, Coady M, et al. Weight lifting and aortic dissection: more evidence for a connection. Cardiology 2007;107:103-6.

25. Rabkin SW. The Role Matrix Metalloproteinases in the Production of Aortic Aneurysm. Prog Mol Biol Transl Sci 2017;147:239-65.

26. Takagi H, Hari Y, Nakashima K, et al. Matrix metalloproteinases and acute aortic dissection: $\mathrm{Et} \mathrm{Tu}$, Brute? Interact Cardiovasc Thorac Surg 2020;30:465-76.

(English Language Editor: K. Brown)

Cite this article as: Zhou H, Ren Y, Xiao J, He J, Zhang Y, Qiu Z, Huang Q, Hu Y, Chen L. Changes in aortic collagen in $\beta$-aminopropionitrile-induced acute aortic dissection. Ann Transl Med 2021;9(20):1574. doi: 10.21037/atm-21-4933 